\title{
A finite difference method for an initial-boundary value problem with a Riemann-Liouville-Caputo spatial fractional derivative
}

\author{
José Luis Gracia ${ }^{1}$, Martin Stynes ${ }^{b}$, \\ ${ }^{a}$ IUMA and Department of Applied Mathematics, University of Zaragoza, Spain. \\ ${ }^{b}$ Applied and Computational Mathematics Division, Beijing Computational Science Research \\ Center, Haidian District, Beijing 100193, P.R.China.
}

\begin{abstract}
An initial-boundary value problem with a Riemann-Liouville-Caputo space fractional derivative of order $\alpha \in(1,2)$ is considered, where the boundary conditions are reflecting. A fractional Friedrichs' inequality is derived and is used to prove that the problem approaches a steady-state solution when the source term is zero. The solution of the general problem is approximated using a finite difference scheme defined on a uniform mesh and the error analysis is given in detail for typical solutions which have a weak singularity near the spatial boundary $x=0$. It is proved that the scheme converges with first order in the maximum norm. Numerical results are given that corroborate our theoretical results for the order of convergence of the difference scheme, the approach of the solution to steady state, and mass conservation.
\end{abstract}

Keywords: Fractional differential equation, time-dependent problem, Riemann-Liouville-Caputo fractional derivative, weak singularity, discrete comparison principle, finite difference scheme, steady-state problem

\section{Introduction}

The problem considered in this paper is inspired by [1], which gives a lengthy discussion of various types of fractional initial-boundary value problem and the boundary conditions that are appropriate for each type. In particular, we shall focus on the "Caputo fractional flux" and reflecting boundary conditions of $[1$, Section $6]$.

\footnotetext{
${ }^{*}$ Corresponding author: m.stynes@ csrc.ac.cn
} 
Set $\Omega:=(0, L)$ and $Q:=\Omega \times(0, T]$. For $(x, t) \in \Omega$ and constant $r>0$, define the Riemann-Liouville integral operator $I_{x}^{r}$ of order $r$ by

$$
I_{x}^{r} v(x, t):=\frac{1}{\Gamma(r)} \int_{s=0}^{x}(x-s)^{r-1} v(s, t) d s .
$$

Then for any positive constant $\beta$ with $n-1<\beta<n$ where $n$ is a positive integer, the Caputo fractional derivative of order $\beta$ is defined by

$$
D_{C, x}^{\beta} v(x, t):=I_{x}^{n-\beta} \frac{\partial^{n} v}{\partial x^{n}}(x, t) .
$$

Here we assume that the function $v$ is such that the definitions make sense.

Let $\alpha$ be constant with $1<\alpha<2$. In this paper, we examine the initialboundary value problem

$$
\begin{aligned}
u_{t}-D_{R L C, x}^{\alpha} u & =f \text { for }(x, t) \in Q, \\
u(x, 0) & =\phi(x) \text { for } x \in \Omega, \\
D_{C, x}^{\alpha-1} u(0, t) & =D_{C, x}^{\alpha-1} u(L, t)=0 \text { for } t \in(0, T],
\end{aligned}
$$

where $D_{R L C, x}^{\alpha}$ is the Riemann-Liouville-Caputo fractional derivative of order $\alpha$, which is defined by

$$
D_{R L C, x}^{\alpha} u(x, t):=\frac{\partial}{\partial x} D_{C, x}^{\alpha-1} u(x, t) \text { for } x>0 \text { and } 0<t \leq T .
$$

This hybrid fractional derivative $D_{R L C, x}^{\alpha}$ has been suggested by several researchers, from both modelling and mathematical viewpoints; see [8] for references. In [1] the quantity $D_{C, x}^{\alpha-1} u$ is called the Caputo fractional flux.

The left boundary condition in (1c) is defined by

$$
0=D_{C, x}^{\alpha-1} u(0, t):=\lim _{x \rightarrow 0^{+}} D_{C, x}^{\alpha-1} u(x, t) .
$$

It is suitable for certain physical models [3] and removes a troublesome singularity from the solution $u(x, t)$ at $x=0$; see $[1,5,8]$ and Remark 1 below. Furthermore, both boundary conditions in (1c) are reflecting and ensure that mass is conserved; see the discussion in [1].

Remark 1. In [8] it is shown that $D_{C, x}^{\alpha-1} u(0, t)=0$ in (1c) is equivalent to the classical Neumann boundary condition $u_{x}(0, t)=0$ if $D_{R L C, x}^{\alpha} u(\cdot, t) \in C[0, L]$. Our analysis in Sections 3 and 4 assumes that the solution $u$ of problem (1) has this regularity, so for convenience our numerical method will discretise $u_{x}(0, t)=0$ 
instead of $D_{C, x}^{\alpha-1} u(0, t)=0$; but the other boundary condition $D_{C, x}^{\alpha-1} u(L, t)=0$ of (1c) cannot be simplified in the same way and must be handled directly.

Remark 2. For the function $x^{\beta}$ with $\alpha-1<\beta<1$, one has [4, p. 193]

$$
D_{C, x}^{\alpha-1} x^{\beta}=\frac{\Gamma(\beta+1)}{\Gamma(\beta-\alpha+2)} x^{\beta-\alpha+1} \text { and } \frac{d}{d x} x^{\beta}=\beta x^{\beta-1} .
$$

Here $\beta-\alpha+1>0$ while $\beta-1<0$, i.e., for these functions $x^{\beta}$, the boundary condition $D_{C, x}^{\alpha-1} u(0, t)=0$ of (1c) does not imply the Neumann boundary condition $u_{x}=0$, unlike the situation described in Remark 1. Thus replacing $D_{C, x}^{\alpha-1} u(0, t)=0$ by $u_{x}(x, 0)=0$ means we exclude functions $u(x, t)$ that behave like a multiple of $x^{\beta}$ as $x \rightarrow 0$ for some fixed value of $t$. But for such functions, in (1a) one has $D_{R L C, x}^{\alpha} u(x, t) \sim O\left(x^{\beta-\alpha}\right)$ near $x=0$ with $\beta-\alpha<0$, so we are merely excluding certain singularities in the terms of the differential equation.

In [2, Proposition 19] it is proved that problem (1) with $f \equiv 0$ is well-posed in the Banach space $L^{1}(0, L)$ for each value of $t$. We shall assume a reasonable amount of smoothness of the solution - see equation (17) below. Our aim is to approximate the solution $u$ of problem (1) by a finite difference method whose analysis requires these bounds on derivatives.

The structure of the paper is as follows. In Section 2 we discuss some properties of the solution $u$ of problem (1). A fractional Friedrichs' inequality for Caputo fractional derivatives is established and is used to prove that $u$ converges to the steady state solution when $f \equiv 0$. A finite difference scheme for solving (1) on a uniform mesh is defined in Section 3 and it is shown that it satisfies a discrete comparison principle. In Section 4 this principle and an appropriate barrier function are used to prove that the solution of the finite difference scheme converges to $u$ with first order in the discrete maximum norm. Three numerical examples are given in Section 5, to illustrate our theoretical results.

Notation: Denote by $A C[0, L]$ the set of absolutely continuous functions on $[0, L]$ and by $L^{p}(0, L)$ the usual Lebesgue space with norm $\|\cdot\|_{L^{P}(0, L)}$. Throughout the paper, $C$ denotes a generic constant that can depend on the data of the problem (1) but it is independent of the mesh used for its numerical solution. Note that $C$ can take different values in different places.

\section{Some properties of the solution}

In this section we first derive a Friedrichs' inequality for Caputo derivatives (Lemma 1). Hence, in Lemma 2, we prove convergence of the solution $u$ to the 
constant steady-state solution $\left(\int_{x=0}^{L} \phi(x) d x\right) / L$ in the special case when $f \equiv 0$. This lemma implies uniqueness of the solution to (1) and stability of this solution in terms of perturbations of the initial condition; see Corollary 1.

Lemma 1 (Friedrichs' inequality for Caputo derivatives). Let $\beta \in(0,1)$. Suppose that $v \in A C[0, L]$ with $\int_{\Omega} v d x=0$ and $\left\|D_{C, x}^{\beta} v\right\|_{L^{2}(0, L)}<\infty$. Then

$$
\|v\|_{L^{2}(0, L)} \leq \frac{L^{\beta}}{\Gamma(\beta+1)}\left\|D_{C, x}^{\beta} v\right\|_{L^{2}(0, L)} .
$$

Proof. For all $x \in(0, L]$, by [4, Theorem 3.8] one has

$$
z(x):=v(x)-v(0)=\left(I_{x}^{\beta} D_{C, x}^{\beta} v\right)(x)=\left(\omega_{\beta} * D_{C, x}^{\beta} v\right)(x),
$$

where $*$ denotes convolution and $\omega_{\beta}(x):=x^{\beta-1} / \Gamma(\beta)$. Hence, similarly to $[6$, Lemma 2.6], we get

$$
\begin{aligned}
\|z\|_{L^{2}(0, L)} & =\left\|\omega_{\beta} * D_{C, x}^{\beta} v\right\|_{L^{2}(0, L)} \\
& \leq\left\|\omega_{\beta}\right\|_{L^{1}(0, L)}\left\|D_{C, x}^{\beta} v\right\|_{L^{2}(0, L)} \\
& =\frac{L^{\beta}}{\Gamma(\beta+1)}\left\|D_{C, x}^{\beta} v\right\|_{L^{2}(0, L)},
\end{aligned}
$$

using Young's inequality for convolutions. But

$$
\|z\|_{L^{2}(0, L)}^{2}=\int_{x=0}^{L}\left[v^{2}(x)-2 v(0) v(x)+v^{2}(0)\right] d x=\|v\|_{L^{2}(0, L)}^{2}+L v^{2}(0),
$$

since $\int_{\Omega} v d x=0$. Thus, (3) implies (2).

Remark 3. If $v \in A C[0, L]$ and $0<\beta<1$, then $\left\|D_{C, x}^{\beta} v\right\|_{L^{p}(0, L)}<\infty$ for $1 \leq p<1 / \beta$ by [4, Lemma 2.12]. Thus in Lemma 1 the hypothesis $v \in A C[0, L]$ implies the hypothesis $\left\|D_{C, x}^{\beta} v\right\|_{L^{2}(0, L)}<\infty$ when $\beta<1 / 2$.

The next lemma shows that when $f \equiv 0$, the solution of problem (1) converges (in the $L^{2}(0, L)$ sense) to the steady-state solution as $t \rightarrow \infty$. A related result was obtained in [1, Appendix] (see Remark 5 below), but using a very technical argument. Our simpler proof is based on the Friedrichs' inequality of Lemma 1.

Lemma 2. Assume that $f \equiv 0$ in (1). Assume also that the solution of problem (1) satisfies $u(x, \cdot) \in A C[0, T]$ for each $x$ and $u_{x}(\cdot, t) \in A C[0, L]$ for each $t$. Then

$$
\left\|u(x, t)-\frac{1}{L} \int_{x=0}^{L} \phi(x) d x\right\|_{L^{2}(0, L)} \rightarrow 0 \quad \text { as } t \rightarrow \infty .
$$


Proof. Set

$$
v(x, t)=u(x, t)-\frac{1}{L} \int_{x=0}^{L} \phi(x) d x
$$

Then

$$
\begin{aligned}
v_{t}-D_{R L C, x}^{\alpha} v & =0 \text { for }(x, t) \in Q, \\
v(x, 0) & =\phi(x)-\frac{1}{L} \int_{x=0}^{L} \phi(x) d x \text { for } x \in(0, L), \\
D_{C, x}^{\alpha-1} v(0, t) & =D_{C, x}^{\alpha-1} v(L, t)=0 \text { for } t \in(0, T] .
\end{aligned}
$$

Observe that $v$ has the additional property that

$$
\int_{x=0}^{L} v(x, 0) d x=0 .
$$

Note first that mass is conserved, i.e., for each $t$ we have

$$
\int_{x=0}^{L} v(x, t)=\int_{x=0}^{L} v(x, 0)=0
$$

to see this, integrate $v_{t}-D_{R L C, x}^{\alpha} v=0$ over $[0, L] \times[0, t]$ and use (4c) to eliminate the $x$-derivative terms.

Let $t \in[0, T]$ be arbitrary. Let $k$ be a constant that is chosen later. Multiply (4a) by $e^{k t} v(x, t)$ then integrate over $[0, L] \times[0, t]$. Using $e^{k t} v v_{t}=e^{k t}\left(v^{2}\right)_{t} / 2$ and integration by parts in time and in space, we get

$$
\begin{aligned}
0=\frac{1}{2} & \int_{x=0}^{L}\left[e^{k t} v^{2}(x, t)-v^{2}(x, 0)\right] d x-\frac{1}{2} \int_{s=0}^{t} k e^{k s} \int_{x=0}^{L} v^{2}(x, s) d s d x \\
& +\int_{s=0}^{t} e^{k s} \int_{x=0}^{L} v_{x}(x, s) D_{C, x}^{\alpha-1} v(x, s) d x d s .
\end{aligned}
$$

These integrations by parts are justified since by hypothesis $v(x, \cdot) \in A C[0, T]$ for each $x$ and $v_{x}(\cdot, t) \in A C[0, L]$ for each $t$, so $D_{C, x}^{\alpha-1} v(\cdot, t)=I_{x}^{2-\alpha} v_{x}(\cdot, t) \in A C[0, L]$ by [9, Lemma 2.3]. But by [10, Lemma 3.1] one has

$$
\begin{aligned}
\int_{x=0}^{L} v_{x}(x, s) D_{C, x}^{\alpha-1} v(x, s) d x & =\int_{x=0}^{L} v_{x}(x, s)\left(I_{x}^{2-\alpha} v_{x}\right)(x, s) d x \\
& \geq\left[\cos \frac{(2-\alpha) \pi}{2}\right] \int_{x=0}^{L}\left(I_{x}^{1-\alpha / 2} v_{x}\right)^{2}(x, s) d x
\end{aligned}
$$




$$
=\left[\cos \frac{(2-\alpha) \pi}{2}\right] \int_{x=0}^{L}\left(D_{C, x}^{\alpha / 2} v\right)^{2}(x, s) d x .
$$

As $v_{x}(\cdot, t) \in A C[0, L]$ for each $t$, one has $D_{C, x}^{\alpha / 2} v(\cdot, t)=\left(I_{x}^{1-\alpha / 2} v_{x}\right)(\cdot, t) \in C[0, L]$ for each $t$, by [9, Lemma 2.3]. Consequently $\left\|D_{C, x}^{\alpha / 2} v(\cdot, t)\right\|_{L^{2}(0, L)}<\infty$. Recalling (5), we can apply Lemma 1 with $\beta=\alpha / 2$ to $v(x, s)$ for each $s$. This gives

$$
\int_{x=0}^{L} v^{2}(x, s) d x \leq \frac{L^{\alpha}}{[\Gamma(1+\alpha / 2)]^{2}} \int_{x=0}^{L}\left(D_{C, x}^{\alpha / 2} v\right)^{2}(x, s) d x .
$$

Now choose

$$
k=2\left[\cos \frac{(2-\alpha) \pi}{2}\right] \frac{[\Gamma(1+\alpha / 2)]^{2}}{L^{\alpha}} .
$$

Then (7), (8) and (9) yield

$$
\frac{k}{2} \int_{x=0}^{L} v^{2}(x, s) d x \leq \int_{x=0}^{L} v_{x}(x, s) D_{C, x}^{\alpha-1} v(x, s) d x
$$

for each $s$. Substituting this inequality into (6) gives

$$
\frac{1}{2} e^{k t} \int_{x=0}^{L} v^{2}(x, t) d x \leq \frac{1}{2} \int_{x=0}^{L} v^{2}(x, 0) d x \text { for each } t,
$$

i.e.,

$$
\|v(\cdot, t)\|_{L^{2}(0, L)} \leq e^{-k t / 2}\|v(\cdot, 0)\|_{L^{2}(0, L)} \text { for each } t .
$$

Thus $\|v(\cdot, t)\|_{L^{2}(0, L)}$ decays exponentially to 0 as $t \rightarrow \infty$, and we are done.

Remark 4. Suppose that $L=1$. From its definition in (9), we see that $k$ increases as $\alpha$ increases (for $1<\alpha<2$ ). Then inequality (10) in our proof of Lemma 2 hints that an increase in $\alpha$ will speed up the convergence of the solution to steady state. This surmise is borne out later by our numerical Example 3 of Section 5.

Remark 5. In [1, Appendix] it is shown that if in (1) with $f \equiv 0$ the boundary condition at $x=0$ is the absorbing condition $u(0, t)=0$ and at $x=L$ one has $u(L, t)=0$ or $D_{C, x}^{\alpha-1}(L, t)=0$ for $0<t \leq T$, then the solution $u(x, t)$ converges (in the sense of $L^{p}(0, L)$ with $\left.1 \leq p<\infty\right)$ exponentially fast to zero as $t \rightarrow \infty$. Our proofs of Lemmas 1 and 2, with very slight modifications, yield this result for $L^{2}(0, L)$. Hölder's inequality then implies the result also for $L^{p}(0, L)$ when $1 \leq p \leq 2$.

The result is also true if $u(L, t)=0$ and either $u(0, t)=0$ or $D_{C, x}^{\alpha-1}(0, t)=0$ for $0<t \leq T$; the proof is essentially similar to the case above - one exploits the 
adjoint property [6, Property A.2] of the left and right Riemann-Liouville fractional integrals in $L^{2}(0, L)$.

Corollary 1 (Stability and uniqueness of solution). Let $u_{1}$ and $u_{2}$ be two solutions of (1) with respective initial conditions $\phi_{1}$ and $\phi_{2}$. Assume that $u_{1}$ and $u_{2}$ satisfy the hypotheses of Lemma 2. Then

$$
\left\|\left(u_{1}-u_{2}\right)(\cdot, t)\right\|_{L^{2}(0, L)} \leq e^{-k t / 2}\left\|\phi_{1}-\phi_{2}\right\|_{L^{2}(0, L)} \text { for } t \in[0, T] .
$$

In addition, if problem (1) has a solution, then the solution is unique.

Proof. Inequality (11) is a consequence of (10). Uniqueness of the solution of (1) follows immediately.

\section{Finite difference scheme}

We shall use a uniform mesh on $[0, L] \times[0, T]$. Let $M$ and $N$ be positive integers. Set $h=L / N$ and $\tau=T / N$. Set $x_{j}=j h$ for $j=0,1, \ldots, M$ and $t_{j}=j \tau$ for $j=0,1, \ldots, N$.

To discretise the spatial derivative in (1a) we follow [8], where the two-point boundary value problem corresponding to (1a) was considered. The time derivative in (1a) is discretised by the backward Euler method.

To discretise the boundary condition (1c) at $x=0$, recall from [8, Section 2] that $D_{C, x}^{\alpha-1} u(0, t)=0$ implies that $u_{x}(0, t)=0$ if $D_{R L C, x}^{\alpha} u(\cdot, t) \in C[0, L]$. From now on, we assume that the solution $u$ of problem (1) has this regularity, and then the boundary condition $u_{x}(0, t)=0$ is approximated by a 2-point divided difference.

Our finite difference scheme is:

$$
\begin{aligned}
L_{M, N} u_{m}^{n} & :=D_{t}^{-} u_{m}^{n}-D_{x}^{+} D_{L_{1}, x}^{\alpha-1} u_{m}^{n}=f\left(x_{m}, t_{n}\right) \text { for } 0<m<M, 0<n \leq N, \\
u_{m}^{0} & =\phi\left(x_{m}\right) \text { for } 0 \leq m \leq M, \\
-D_{x}^{+} u_{0}^{n} & =0, D_{L_{1}, x}^{\alpha-1} u_{M}^{n}=0 \text { for } 0<n \leq N,
\end{aligned}
$$

where $u_{m}^{n}$ is the solution of (12) at the point $\left(x_{m}, t_{n}\right)$. In (12a) and (12c), the operators $D_{t}^{-}$and $D_{x}^{+}$denote backward and forward divided differences:

$$
D_{t}^{-} Z_{m}^{n}:=\frac{Z_{m}^{n}-Z_{m}^{n-1}}{\tau}, \quad D_{x}^{+} Z_{m}^{n}:=\frac{Z_{m+1}^{n}-Z_{m}^{n}}{h} .
$$


In (12c), $D_{L_{1}, x}^{\alpha-1}$ is the standard $L 1$ discretisation of the Caputo derivative $D_{C, x}^{\alpha-1}$, viz.,

$$
\begin{aligned}
D_{L 1, x}^{\alpha-1} Z_{m}^{n} & :=\frac{1}{h^{\alpha-1} \Gamma(3-\alpha)} \sum_{k=0}^{m-1}\left(Z_{k+1}^{n}-Z_{k}^{n}\right) d_{m-k} \\
& =\frac{1}{h^{\alpha-1} \Gamma(3-\alpha)}\left[Z_{m}^{n} d_{1}-Z_{0}^{n} d_{m}+\sum_{k=1}^{m-1} Z_{k}^{n}\left(d_{m-k+1}-d_{m-k}\right)\right],
\end{aligned}
$$

for $m=1,2, \ldots, M$, where $d_{k}:=(2-\alpha) \int_{s=k-1}^{k} s^{1-\alpha} d s$ for $k=1,2, \ldots, M$.

Thus,

$$
\begin{aligned}
-D_{x}^{+}\left(D_{L 1, x}^{\alpha-1} Z_{m}^{n}\right) & =\frac{1}{h}\left(D_{L 1, x}^{\alpha-1} Z_{m+1}^{n}-D_{L 1, x}^{\alpha-1} Z_{m}^{n}\right) \\
& =-\frac{1}{h^{\alpha} \Gamma(3-\alpha)}\left[\sum_{k=0}^{m}\left(Z_{k+1}^{n}-Z_{k}^{n}\right) d_{m+1-k}-\sum_{k=0}^{m-1}\left(Z_{k+1}^{n}-Z_{k}\right) d_{m-k}\right] \\
& =-\frac{1}{h^{\alpha} \Gamma(3-\alpha)}\left[\left(Z_{1}^{n}-Z_{0}^{n}\right) d_{m+1}+\sum_{k=0}^{m-1}\left(Z_{k+2}^{n}-2 Z_{k+1}^{n}+Z_{k}^{n}\right) d_{m-k}\right] \\
& =-\frac{1}{h^{\alpha} \Gamma(3-\alpha)}\left[\left(Z_{1}^{n}-Z_{0}^{n}\right) d_{m+1}\right]-D_{L 2, x}^{\alpha} Z_{m}^{n},
\end{aligned}
$$

where $D_{L 2, x}^{\alpha}$ is the L2 discretization [12, equation (4.1)] of the Caputo fractional derivative $D_{C, x}^{\alpha} u\left(x_{n}, t_{m}\right)$. Rearranging terms, one has

$$
\begin{aligned}
-D_{x}^{+}\left(D_{L 1, x}^{\alpha-1} Z_{m}^{n}\right)=- & \frac{1}{h^{\alpha} \Gamma(3-\alpha)}\left[Z_{m+1}^{n} d_{1}+Z_{m}^{n}\left(d_{2}-2 d_{1}\right)\right. \\
& \left.+Z_{0}^{n}\left(d_{m}-d_{m+1}\right)+\sum_{k=1}^{m-1} Z_{k}^{n}\left(d_{m-k+2}-2 d_{m-k+1}+d_{m-k}\right)\right] .
\end{aligned}
$$

We shall prove that the scheme (12) satisfies a discrete comparison principle. This property will be used to analyse the convergence of its solution $\left\{u_{m}^{n}\right\}$ to the solution $u$ of problem (1).

Lemma 3 (Discrete comparison principle). Let $\left\{Z_{m}^{n}\right\}_{m=0, n=0}^{M, N}$ be a mesh function. If

$$
\begin{aligned}
Z_{m}^{0} & \geq 0 \text { for } 0 \leq m \leq M, \\
-D^{+} Z_{0}^{n} & \geq 0 \text { and } D_{L 1, x}^{\alpha-1} Z_{M}^{n} \geq 0 \text { for } 0<n \leq N, \\
L_{M, N} Z_{m}^{n} & \geq 0 \text { for } 0<m<M \text { and } 0<n \leq N,
\end{aligned}
$$

then $Z_{m}^{n} \geq 0$ for $0 \leq m \leq M$ and $0 \leq n \leq N$. 
Proof. Set $Z^{n}=\left(Z_{0}^{n}, \ldots, Z_{M}^{n}\right)^{T}$ for each $n$. We use induction on $n$ (cf. [11, Lemma 3.12]) to prove that $Z^{n} \geq 0$, i.e., that $Z_{m}^{n} \geq 0$ for each $m$. By hypothesis $Z^{0} \geq 0$. Assume that $Z^{n} \geq 0$ for some $n \in\{0,1, \ldots, N-1\}$; we wish to prove that $Z^{n+1} \geq 0$. The inequalities $L_{M, N} Z_{m}^{n+1} \geq 0$ for $0<m<M,-D^{+} Z_{0}^{n+1} \geq 0$ and $D_{L 1, x}^{\alpha-1} Z_{M}^{n+1} \geq 0$ can be written in the form

$$
L_{M, N} Z^{n+1}=A Z^{n+1}-B Z^{n} \geq 0
$$

where $A$ is a lower Hessenberg matrix whose entries are given below, and $B$ is the diagonal matrix with diagonal entries $\{0,1 / \tau, 1 / \tau, \ldots, 1 / \tau, 0\}$. By the induction hypothesis, we have

$$
A Z^{n+1} \geq 0 .
$$

We now prove that $A^{-1}>0$. The entries of the matrix $A=\left(a_{i j}\right)_{i, j=0}^{M}$ associated with the boundary condition at $x=0$ are

$$
a_{00}=1 / h, a_{01}=-1 / h, a_{0 j}=0 \text { for } j=2,3, \ldots, M-1 .
$$

For $0<i<M$, recalling (14), the entries of the $i$ th row of the matrix $A$ are

$$
\begin{aligned}
a_{i 0} & =\frac{d_{i+1}-d_{i}}{h^{\alpha} \Gamma(3-\alpha)}<0 \\
a_{i j} & =\frac{-d_{i-j}+2 d_{i-j+1}-d_{i-j+2}}{h^{\alpha} \Gamma(3-\alpha)}<0 \text { for } j=1,2, \ldots, i-1, \\
a_{i i} & =\frac{1}{\tau}+\frac{2 d_{1}-d_{2}}{h^{\alpha} \Gamma(3-\alpha)}>0, a_{i, i+1}=\frac{-d_{1}}{h^{\alpha} \Gamma(3-\alpha)}<0, \\
a_{i j} & =0 \text { for } j=i+2, i+3, \ldots, M
\end{aligned}
$$

(the second inequality, $a_{i j}<0$, is verified in [12, Section 4.1]). If $i=M$, then (13) yields

$$
\begin{aligned}
a_{M 0} & =-\frac{d_{M}}{h^{\alpha-1} \Gamma(3-\alpha)}<0, \quad a_{M j}=\frac{d_{M-j+1}-d_{M-j}}{h^{\alpha-1} \Gamma(3-\alpha)}<0 \text { for } j=1,2, \ldots, M-1, \\
a_{M M} & =\frac{d_{1}}{h^{\alpha-1} \Gamma(3-\alpha)}>0 .
\end{aligned}
$$

Thus, the diagonal entries of $A$ are positive and its off-diagonal entries are nonpositive. Furthermore, one has

$$
\sum_{j=0}^{M} a_{0 j}=0, \quad \sum_{j=0}^{M} a_{i j}=\frac{1}{\tau}>0 \text { for } 0<i<M, \quad \sum_{j=0}^{M} a_{M j}=0 .
$$


It follows that $A$ is an irreducibly diagonal dominant matrix [14, p.23]. Now [14, Corollary 3.20, p.91] gives $A^{-1}>0$. Thus, recalling (15), we get $Z^{n+1} \geq 0$. This completes the inductive step and the proof.

\section{Error estimate for scheme}

In this section an error bound for our difference scheme is derived. First, in (17) we make an assumption on the behaviour of the derivatives of the solution $u$ that is motivated by considering the behaviour of steady-state solutions. This enables truncation error bounds to be established for the scheme; see Section 4.2. To convert these bounds to an error estimate for the computed solution, we shall employ a barrier function whose construction is discussed in Section 4.3. Finally, in Theorem 1, our error bound is obtained.

\subsection{Bounds on derivatives}

To prove convergence of the scheme (12), one needs a priori bounds on certain derivatives of $u$. We shall assume these bounds in (17) below. To motivate them, consider first a particular case of the steady version of the problem (1):

$$
\begin{aligned}
-D_{R L C}^{\alpha} v(x)+b v(x) & =\sum_{i=0}^{\ell} \frac{a_{i}}{i !} x^{i} \text { for } x \in(0, L), \\
D_{C}^{\alpha-1} v(0) & =0, D_{C}^{\alpha-1} v(L)=0,
\end{aligned}
$$

with constants $a_{i}$ for $i=0,1, \ldots, \ell$, constant $b>0$ and $\sum_{i=0}^{\ell}\left|a_{i}\right| \neq 0$ (as otherwise $v \equiv 0$.) By means of Laplace transforms, one finds that the solution of this problem is (see [8] for a related calculation)

$$
v(x)=\left(\frac{\sum_{i=0}^{\ell} a_{i} L^{i} E_{\alpha, 2+i}\left(b L^{\alpha}\right)}{b E_{\alpha, 2}\left(b L^{\alpha}\right)}\right) E_{\alpha, 1}\left(b x^{\alpha}\right)-\sum_{i=0}^{\ell} a_{i} x^{\alpha+i} E_{\alpha, 1+i+\alpha}\left(b x^{\alpha}\right),
$$

where $E_{\delta, \gamma}(z)$, for $\delta, \gamma, z \in \mathbb{R}$ and $\delta>0$, denotes the two-parameter Mittag-Leffler function defined by

$$
E_{\delta, \gamma}(z)=\sum_{k=0}^{\infty} \frac{z^{k}}{\Gamma(\delta k+\gamma)}
$$


If $\ell=0$, the solution of (16) is constant with $v \equiv a_{0} / b$. Otherwise,

$$
\begin{aligned}
v(x)=\frac{\sum_{i=0}^{\ell} a_{i} L^{i} E_{\alpha, 2+i}\left(b L^{\alpha}\right)}{b E_{\alpha, 2}\left(b L^{\alpha}\right)}+\frac{x^{\alpha}}{\Gamma(1+\alpha)}\left(\frac{\sum_{i=0}^{\ell} a_{i} L^{i} E_{\alpha, 2+i}\left(b L^{\alpha}\right)}{E_{\alpha, 2}\left(b L^{\alpha}\right)}-a_{0}\right) \\
+ \text { higher-order terms. }
\end{aligned}
$$

One sees that the derivatives of $v$ satisfy $\left|v^{(i)}(x)\right| \leq C x^{\alpha-i}$ for $i=1,2, \ldots$.

A similar bound is proved in [8, Corollary 2.1], where a two-point boundary value problem with variable coefficients is examined, but with a different boundary condition at $x=L$. This alteration of the boundary condition at $x=L$ should not modify significantly the behaviour of the derivatives of the solution, since any loss of regularity occurs near $x=0$.

For the time-dependent problem (1), one expects that the classical time derivative will not affect the behaviour of the spatial derivatives. Thus, we assume the following bounds on the derivatives of the solution:

$$
\left|\frac{\partial^{j} u}{\partial t^{j}}(x, t)\right| \leq C \text { for } j=0,1,2 \text { and }\left|\frac{\partial^{i} u}{\partial x^{i}}(x, t)\right| \leq C x^{\alpha-i} \text { for } i=1,2,3,
$$

for all $(x, t) \in Q$.

\subsection{Truncation error estimates}

It is easy to see that $\left|D_{t}^{-} u\left(x_{m}, t_{n}\right)-u_{t}\left(x_{m}, t_{n}\right)\right| \leq C \tau$. Next, [8, Lemma 4.1] gives

$$
\begin{gathered}
\left|L_{M, N}\left(u_{m}^{n}-u\left(x_{m}, t_{n}\right)\right)\right| \leq C h x_{m}^{-1}+C \tau, \\
\left|D_{x}^{+}\left(u\left(0, t_{n}\right)-u_{0}^{n}\right)\right| \leq C h^{\alpha-1} .
\end{gathered}
$$

It remains to bound the truncation error at $x_{M}=L$.

Lemma 4. Assume that the derivatives of the solution $u$ of the problem (1) satisfy (17). Then there exists a constant $C$ such that

$$
\left|D_{L 1, x}^{\alpha-1} u\left(x_{M}, t_{n}\right)-D_{C, x}^{\alpha-1} u\left(x_{M}, t_{n}\right)\right| \leq C h^{\min \{\alpha, 3-\alpha\}} \leq C h \text { for } n=1,2, \ldots, N .
$$

Proof. The truncation error for this term is

$$
D_{L 1, x}^{\alpha-1} u\left(x_{M}, t_{n}\right)-D_{C, x}^{\alpha-1} u\left(x_{M}, t_{n}\right)=\sum_{k=0}^{M-1} T_{M k},
$$


where

$$
T_{M k}:=\frac{1}{\Gamma(2-\alpha)} \int_{s=x_{k}}^{x_{k+1}}\left(x_{M}-s\right)^{1-\alpha}\left[\frac{u\left(x_{k+1}, t_{n}\right)-u\left(x_{k}, t_{n}\right)}{h}-\frac{\partial u}{\partial s}\left(s, t_{n}\right)\right] d s .
$$

Proceeding similarly to the derivation of [13, equations (5.13) and (5.14)] but using (17), we get

$$
\left|T_{M 0}\right| \leq C h^{\alpha} \text { and }\left|T_{M, M-1}\right| \leq C h^{3-\alpha} .
$$

If $0<k<M-1$, by imitating [13, equation (5.6)] one has

$$
\begin{aligned}
\left|T_{M k}\right| & \leq C h^{2}\left(\max _{s \in\left[x_{k}, x_{k+1}\right]}\left|u_{x x}\left(s, t_{n}\right)\right|\right) \int_{s=x_{k}}^{x_{k+1}}\left(x_{M}-s\right)^{-\alpha} d s \\
& \leq C h^{3} x_{k}^{\alpha-2}\left(x_{m}-x_{k+1}\right)^{-\alpha} \\
& \leq C h k^{\alpha-2}[M-(k+1)]^{-\alpha},
\end{aligned}
$$

from (17). Using this bound for $\left|T_{M k}\right|$ like the derivation of [13, equations (5.9) and (5.10)], one obtains

$$
\sum_{k=1}^{\lceil M / 2\rceil-1}\left|T_{M k}\right| \leq C h^{2} \text { and } \sum_{k=\lceil M / 2\rceil-1}^{M-2}\left|T_{M k}\right| \leq C h^{3-\alpha} .
$$

Adding all these bounds, we get

$$
\left|D_{L 1, x}^{\alpha-1} u\left(x_{M}, t_{n}\right)-D_{C, x}^{\alpha-1} u\left(x_{M}, t_{n}\right)\right| \leq C h^{\min \{\alpha, 3-\alpha\}} \leq C h .
$$

\subsection{Discrete barrier function}

To obtain a bound on the error in the solution computed by the finite difference method (12), we shall use a barrier function. That is, on the grid $\left\{\left(x_{m}, t_{n}\right)\right\}_{m, n=0}^{M, N}$ we construct a non-negative discrete function $\left\{\widetilde{\Psi}_{m}^{n}\right\}_{m=0, n=0}^{M, N}$ that satisfies

$$
\begin{aligned}
\left|L_{M, N}\left(u\left(x_{m}, t_{n}\right)-u_{m}^{n}\right)\right| & \leq L_{M, N} \widetilde{\Psi}_{m}^{n} \text { for } 0<m<M \text { and } 0<n \leq N, \\
\left|-D_{x}^{+}\left(u\left(0, t_{n}\right)-u_{0}^{n}\right)\right| & \leq-D_{x}^{+} \widetilde{\Psi}_{0}^{n} \text { for } 0<n \leq N \\
\left|D_{L 1, x}^{\alpha-1}\left(u\left(L, t_{n}\right)-u_{M}^{n}\right)\right| & \leq D_{L 1, x}^{\alpha-1} \widetilde{\Psi}_{M}^{n} \text { for } 0<n \leq N .
\end{aligned}
$$

Then, applying Lemma 3 (discrete comparison principle) to $\widetilde{\Psi}_{m}^{n} \pm\left(u_{m}^{n}-u\left(x_{m}, t_{n}\right)\right)$, one gets

$$
\left|u_{m}^{n}-u\left(x_{m}, t_{n}\right)\right| \leq \widetilde{\Psi}_{m}^{n} \text { for all } m \text { and } n .
$$


The mesh function $\left\{\widetilde{\Psi}_{m}^{n}\right\}_{m=0, n=0}^{M, N}$ is called a discrete barrier function.

To construct this discrete barrier function, we start from [8, Section 4], where the following discrete barrier function was used (for a two-point boundary value problem equivalent to our steady-state problem, but with a different boundary condition at $x=L$ ):

$$
\Psi_{m}=C_{1} h|\ln h|\left(L^{|\ln h|^{-1}+\alpha-1}-T_{m}\right) \text { for } 0 \leq m \leq M,
$$

where $C_{1}$ is a user-chosen constant and $\left\{T_{m}\right\}_{m=0}^{M}$ is defined by

$$
D_{L 1, x}^{\alpha-1} T_{m}=\frac{1}{\Gamma(2-\alpha)} x_{m}^{|\ln h|^{-1}} \text { for } m \geq 1, \quad T_{0}=0 .
$$

Now $0 \leq T_{m} \leq x_{m}^{\left.\ln h\right|^{-1}+\alpha-1}$ for $0 \leq m \leq M$ (see [8, equation (4.14)]), so (19) implies that $\Psi_{m} \geq 0$ for $0 \leq m \leq M$. In addition, in [8] it is proved that

$$
\begin{aligned}
-D_{x}^{+} \Psi_{0} & =C_{1}(2-\alpha) e^{-1} h^{\alpha-1}|\ln h|, \\
-D_{x}^{+} D_{L 1, x}^{\alpha-1} \Psi_{m} & \geq \frac{C_{1} h e^{-1}}{2 \Gamma(2-\alpha)} x_{m}^{-1}, \\
D_{L 1, x}^{\alpha-1} \Psi_{M} & =-\frac{C_{1} h|\ln h|}{\Gamma(2-\alpha)} .
\end{aligned}
$$

But $D_{L 1, x}^{\alpha-1} \Psi_{M}<0$, so this mesh function cannot satisfy condition (18c). Thus, we shall modify it by adding another mesh function $\left\{B_{m}^{n}\right\}_{m=0, n=0}^{M, N}$ to deal with the boundary condition at $x=L$ and also the term $\left|D_{t}^{-} u\left(x_{m}, t_{n}\right)-u_{t}\left(x_{m}, t_{n}\right)\right|$, in order that (18a), (18b) and (18c) are all satisfied.

Define $\left\{R_{m}\right\}_{m=0}^{M}$ by

$$
D_{L 1, x}^{\alpha-1} R_{m}=\left(L-\alpha x_{m}\right) \Gamma(\alpha) \text { for } m=1,2, \ldots, M, \quad R_{0}=0 .
$$

Since $D_{C, x}^{\alpha-1}\left(L x^{\alpha-1}-x^{\alpha}\right)=(L-\alpha x) \Gamma(\alpha)$, we see that $\left\{R_{m}\right\}_{m=0}^{M}$ is the approximation to $\left\{L x_{m}^{\alpha-1}-x_{m}^{\alpha}\right\}$ that is computed by the L1 scheme. Hence, an inspection of [13, Lemma 5.2 and Theorem 5.3] shows that

$$
\left|R_{m}-\left(L x_{m}^{\alpha-1}-x_{m}^{\alpha}\right)\right| \leq C h^{\alpha-1} \text { for all } m,
$$

where $C$ is independent of $M$. It follows that

$$
\max _{0 \leq m \leq M}\left|R_{m}\right| \leq C_{2}
$$

where the constant $C_{2}$ is independent of $M$. 
Consider the mesh function

$$
B_{m}^{n}:=C_{3}(\tau+h|\ln h|)\left(1+t_{n}\right)-C_{4} h|\ln h| R_{m}
$$

where $C_{3}$ and $C_{4}$ are positive constants that we shall specify later. Define

$$
\widetilde{\Psi}_{m}^{n}:=\Psi_{m}+B_{m}^{n}=C_{1} h|\ln h|\left(L^{|\ln h|^{-1}+\alpha-1}-T_{m}\right)+B_{m}^{n} \text { for all } m \text { and } n .
$$

This mesh function will be our barrier function.

\subsection{Error bound for scheme}

We can now prove an error estimate for the solution computed by our scheme.

Theorem 1. Assume that the derivatives of the solution $u$ of the problem (1) satisfy (17). Assume that $h<L / \alpha$. Choose the constants $C_{3}$ and $C_{4}$ to satisfy

$$
C_{4}>\frac{C_{1}}{L(\alpha-1) \Gamma(2-\alpha) \Gamma(\alpha)} \text { and } C_{3} \geq C_{4} \max \left\{\Gamma(\alpha+1), C_{2}\right\} .
$$

Then there exists a constant $C$ such that

$$
\max _{\substack{0 \leq m \leq M \\ 0 \leq n \leq N}}\left|u_{m}^{n}-u\left(x_{m}, t_{n}\right)\right| \leq C(\tau+h|\ln h|),
$$

where $\left\{u_{m}^{n}\right\}_{m=0, n=0}^{M, N}$ is the solution of the finite difference scheme (12).

Proof. To prove that $\left\{\widetilde{\Psi}_{m}^{n}\right\}_{m=0, n=0}^{M, N}$ is a barrier function for the errors $\left\{\left|u_{m}^{n}-u\left(x_{m}, t_{n}\right)\right|\right\}$, note first that (24) implies that

$$
B_{m}^{n} \geq h|\ln h|\left(C_{3}-C_{4} R_{m}\right) \geq 0 \text { for all } m \text { and } n .
$$

As $\Psi_{m} \geq 0$, clearly $\widetilde{\Psi}_{m}^{n}=\Psi_{m}+B_{m}^{n} \geq 0$ for all $m$ and $n$.

Consider the boundary condition at $x=L$. From (23) and $1<\alpha<2$ we have $D_{L 1, x}^{\alpha-1} R_{M}=L(1-\alpha) \Gamma(\alpha)<0$. Hence, $D_{L 1, x}^{\alpha-1} B_{M}^{n}=-C_{4} h|\ln h| D_{L 1, x}^{\alpha-1} R_{M}>0$, and

$$
D_{L 1, x}^{\alpha-1} \widetilde{\Psi}_{M}^{n}=D_{L 1, x}^{\alpha-1} \Psi_{M}+D_{L 1, x}^{\alpha-1} B_{M}^{n}=h|\ln h|\left[C_{4} L(\alpha-1) \Gamma(\alpha)-\frac{C_{1}}{\Gamma(2-\alpha)}\right]>0,
$$

from the choice of $C_{4}$ in (24).

Now consider the boundary condition at $x=0$. We need to compute $R_{1}$ in order to ascertain the sign of $D_{x}^{+} B_{0}^{n}$. From the definition of the L1 scheme, one has

$$
D_{L 1, x}^{\alpha-1} R_{1}=\frac{1}{h^{\alpha-1} \Gamma(3-\alpha)}\left(R_{1}-R_{0}\right) d_{1}=\frac{R_{1}}{h^{\alpha-1} \Gamma(3-\alpha)} .
$$


Hence $R_{1}=h^{\alpha-1} \Gamma(3-\alpha) \Gamma(\alpha)(L-\alpha h)>0$, where we used (23). Now

$$
-D_{x}^{+} B_{0}^{n}=C_{4} h|\ln h| \frac{R_{1}-R_{0}}{h}=C_{4}|\ln h| R_{1}>0 .
$$

Using this inequality and (20) yields

$$
-D_{x}^{+} \widetilde{\Psi}_{0}^{n}=-D_{x}^{+} \Psi_{0}-D_{x}^{+} B_{0}^{n} \geq C h^{\alpha-1}|\ln h| .
$$

Finally, at the interior mesh points, recalling the definition of $\left\{R_{m}\right\}_{m=0}^{M}$ in (23), one obtains

$$
\begin{aligned}
L_{M, N} B_{m}^{n} & =C_{3}(\tau+h|\ln h|) \frac{t_{n+1}-t_{n}}{\tau}+C_{4} h|\ln h| D_{x}^{+} D_{L 1, x}^{\alpha-1} R_{m} \\
& =C_{3}(\tau+h|\ln h|)+C_{4} \Gamma(\alpha) h|\ln h| \frac{\left(L-\alpha x_{m+1}\right)-\left(L-\alpha x_{m}\right)}{h} \\
& =C_{3} \tau+h|\ln h|\left(C_{3}-C_{4} \Gamma(\alpha+1)\right) \\
& \geq C_{3} \tau,
\end{aligned}
$$

where (24) was used. From this inequality and (21) one has

$$
L_{M, N} \widetilde{\Psi}_{m}^{n}=L_{M, N} B_{m}^{n}+L_{M, N} \Psi_{m} \geq C_{3} \tau+\frac{C_{1} h e^{-1}}{2 \Gamma(2-\alpha)} x_{m}^{-1} .
$$

That is, the mesh function $\widetilde{\Psi}_{m}^{n}$ satisfies (18a), (18b) and (18c). Consequently

$$
\left|u_{m}^{n}-u\left(x_{m}, t_{n}\right)\right| \leq \widetilde{\Psi}_{m}^{n} \leq C(\tau+h|\ln h|) \text { for all } m \text { and } n,
$$

as desired.

Remark 6. If in the problem (1) a Dirichlet boundary condition were imposed at $x=L$ and in the scheme we set $u_{M}^{n}=u\left(L, t_{n}\right)$ for $n=0,1, \ldots, N$, then the simpler barrier function

$$
\widetilde{\Psi}_{m}^{n}:=C\left(\tau t_{n}+\Psi_{m}\right)
$$

can be used, where $\left\{\Psi_{m}\right\}_{m=0}^{M}$ is defined in (19). One then obtains the error estimate

$$
\max _{\substack{0 \leq m \leq M \\ 0 \leq n \leq N}}\left|u_{m}^{n}-u\left(x_{m}, t_{n}\right)\right| \leq C(\tau+h|\ln h|)
$$

\section{Numerical experiments}

We present numerical results for three examples. In the first example, the exact solution is known and satisfies the bounds (17); its numerical results illustrate the 
error estimates of Theorem 1. In the second example the exact solution is unknown, so bounds on its partial derivatives are not available. Nevertheless, the results show that the scheme (12) is convergent. In the third example, convergence to steady state and mass conservation are discussed.

\section{Example 1. Consider the problem}

$$
\begin{aligned}
u_{t}-D_{R L C, x}^{\alpha} u & =f(x, t) \text { for }(x, t) \in(0,1) \times(0,1], \\
u(x, 0) & =\left(-x^{2}+r x^{\alpha}\right) \text { for } x \in(0,1), \\
D_{C, x}^{\alpha-1} u(0, t) & =D_{C, x}^{\alpha-1} u(1, t)=0 \text { for } t \in(0,1],
\end{aligned}
$$

where $f(x, t)$ is chosen such that

$$
u(x, t)=\left(1+t^{3}\right)\left(-x^{2}+r x^{\alpha}\right),
$$

and the constant $r$ is chosen so that the boundary conditions are satisfied:

$$
D_{C, x}^{\alpha-1} u(x, t)=\left(1+t^{3}\right)\left(\frac{-2}{\Gamma(4-\alpha)} x^{3-\alpha}+r \Gamma(\alpha+1) x\right)
$$

so $D_{C, x}^{\alpha-1} u(0, t)=0$ and $r=\frac{2}{\Gamma(4-\alpha) \Gamma(\alpha+1)}$ yields

$$
D_{C, x}^{\alpha-1} u(1, t)=\left(1+t^{3}\right)\left(\frac{-2}{\Gamma(4-\alpha)}+r \Gamma(\alpha+1)\right)=0 .
$$

In this example the solution $u(x, t)$ is chosen to agree with the bounds on derivatives stated in (17). The solution $u(x, t)$ of Example 1 is approximated by the solution $\left\{u_{m}^{n}\right\}_{m=0, n=0}^{M, N}$ of the scheme (12). The maximum nodal errors are

$$
E_{M, N}:=\max _{\substack{0 \leq m \leq M \\ 0 \leq n \leq N}}\left|u_{m}^{n}-u\left(x_{m}, t_{n}\right)\right|
$$

These values are used to compute the orders of convergence

$$
p_{M, N}:=\log _{2}\left(\frac{E_{M, N}}{E_{2 M, 2 N}}\right) .
$$

In order to compute separate error estimates for the dependence of the error on the spatial and temporal mesh widths, in the numerical experiments we take one of the discretization parameters $(M$ or $N$ ) smaller than the other one ( $N$ or $M)$. The values of $E_{M, N}$ and $p_{M, N}$ are given in Tables 1 and 2 where $N=4 M$ and $M=4 N$, respectively. In both tables one observes that (12) is a first-order 
convergent scheme, which is slightly better than the error estimate of Theorem 1.

Table 1: Example 1: Maximum nodal errors and orders of convergence

\begin{tabular}{|c||c|c|c|c|c|c|}
\hline & $\mathrm{M}=32$ & $\mathrm{M}=64$ & $\mathrm{M}=128$ & $\mathrm{M}=256$ & $\mathrm{M}=512$ & $\mathrm{M}=1024$ \\
& $\mathrm{~N}=128$ & $\mathrm{~N}=256$ & $\mathrm{~N}=512$ & $\mathrm{~N}=1024$ & $\mathrm{~N}=2048$ & $\mathrm{~N}=4096$ \\
\hline$\alpha=1.2$ & $4.164 \mathrm{E}-02$ & $2.109 \mathrm{E}-02$ & $1.063 \mathrm{E}-02$ & $5.337 \mathrm{E}-03$ & $2.675 \mathrm{E}-03$ & $1.340 \mathrm{E}-03$ \\
& 0.982 & 0.989 & 0.993 & 0.996 & 0.998 & \\
\hline$\alpha=1.4$ & $4.254 \mathrm{E}-02$ & $2.178 \mathrm{E}-02$ & $1.106 \mathrm{E}-02$ & $5.590 \mathrm{E}-03$ & $2.815 \mathrm{E}-03$ & $1.414 \mathrm{E}-03$ \\
& 0.966 & 0.977 & 0.985 & 0.990 & 0.993 & \\
\hline$\alpha=1.6$ & $4.042 \mathrm{E}-02$ & $2.126 \mathrm{E}-02$ & $1.104 \mathrm{E}-02$ & $5.679 \mathrm{E}-03$ & $2.901 \mathrm{E}-03$ & $1.474 \mathrm{E}-03$ \\
& 0.927 & 0.945 & 0.959 & 0.969 & 0.977 & \\
\hline$\alpha=1.8$ & $2.987 \mathrm{E}-02$ & $1.641 \mathrm{E}-02$ & $8.861 \mathrm{E}-03$ & $4.722 \mathrm{E}-03$ & $2.489 \mathrm{E}-03$ & $1.301 \mathrm{E}-03$ \\
& 0.864 & 0.889 & 0.908 & 0.924 & 0.936 & \\
\hline \hline
\end{tabular}

Table 2: Example 1: Maximum nodal errors and orders of convergence

\begin{tabular}{|c||c|c|c|c|c|c|}
\hline & $\mathrm{M}=64$ & $\mathrm{M}=128$ & $\mathrm{M}=256$ & $\mathrm{M}=512$ & $\mathrm{M}=1024$ & $\mathrm{M}=2048$ \\
& $\mathrm{~N}=16$ & $\mathrm{~N}=32$ & $\mathrm{~N}=64$ & $\mathrm{~N}=128$ & $\mathrm{~N}=256$ & $\mathrm{~N}=512$ \\
\hline$\alpha=1.2$ & $3.371 \mathrm{E}-02$ & $1.681 \mathrm{E}-02$ & $8.405 \mathrm{E}-03$ & $4.205 \mathrm{E}-03$ & $2.103 \mathrm{E}-03$ & $1.052 \mathrm{E}-03$ \\
& 1.004 & 1.000 & 0.999 & 0.999 & 0.999 & \\
\hline$\alpha=1.4$ & $3.234 \mathrm{E}-02$ & $1.619 \mathrm{E}-02$ & $8.115 \mathrm{E}-03$ & $4.068 \mathrm{E}-03$ & $2.038 \mathrm{E}-03$ & $1.021 \mathrm{E}-03$ \\
& 0.998 & 0.996 & 0.996 & 0.997 & 0.998 & \\
\hline$\alpha=1.6$ & $2.902 \mathrm{E}-02$ & $1.478 \mathrm{E}-02$ & $7.514 \mathrm{E}-03$ & $3.809 \mathrm{E}-03$ & $1.926 \mathrm{E}-03$ & $9.712 \mathrm{E}-04$ \\
& 0.973 & 0.976 & 0.980 & 0.984 & 0.987 & \\
\hline$\alpha=1.8$ & $2.067 \mathrm{E}-02$ & $1.090 \mathrm{E}-02$ & $5.716 \mathrm{E}-03$ & $2.979 \mathrm{E}-03$ & $1.544 \mathrm{E}-03$ & $7.961 \mathrm{E}-04$ \\
& 0.924 & 0.931 & 0.940 & 0.948 & 0.956 & \\
\hline \hline
\end{tabular}

Example 2. Consider the problem

$$
\begin{aligned}
u_{t}-D_{R L C, x}^{\alpha} u & =10 \sin (2 \pi x) \text { for }(x, t) \in(0,1) \times(0,1], \\
u(x, 0) & =-x^{3}+r x^{2} \text { for } x \in(0,1), \\
D_{C, x}^{\alpha-1} u(0, t) & =D_{C, x}^{\alpha-1} u(1, t)=0 \text { for } t \in(0,1],
\end{aligned}
$$

where $r=3 / \Gamma(4-\alpha)$.

Note that $D_{C, x}^{\alpha-1} u(0,0)=D_{C, x}^{\alpha-1} u(1,0)=0$ by the choice of $r$. 
The exact solution of Example 2 is unknown, so we estimate the orders of convergence by means of the two-mesh principle [7]: the scheme (12) is used to compute solutions $\left\{u_{m}^{n}\right\}_{m=0, n=0}^{M, N}$ and $\left\{\hat{u}_{m}^{n}\right\}_{m=0, n=0}^{2 M, 2 N}$ on two uniform meshes $\left\{\left(x_{m}, t_{n}\right)\right\}_{m=0, n=0}^{M, N}$ and $\left\{\left(\hat{x}_{m}, \hat{t}_{n}\right)\right\}_{m=0, n=0}^{2 M, N}$ respectively. Since the meshes are uniform, one has $x_{m}=\hat{x}_{2 m}$ for $m=0,1, \ldots, N$ and $t_{n}=\hat{t}_{2 n}$ for $n=0,1, \ldots, N$. Use these solutions to calculate the maximum two-mesh nodal difference

$$
D_{M, N}:=\max _{\substack{0 \leq m \leq M \\ 0 \leq n \leq N}}\left|u_{m}^{n}-\hat{u}_{2 m}^{2 n}\right|,
$$

then estimate the orders of convergence by

$$
q_{M, N}:=\log _{2}\left(\frac{D_{M, N}}{D_{2 M, 2 N}}\right) .
$$

Tables 3 and 4 display $D_{M, N}$ and $q_{M, N}$ for $\alpha=1.2,1.4,1.6$ and 1.8. We take $N=4 M$ in Table 3 so that the two-mesh nodal differences associated with the spatial mesh width dominate the errors associated with the temporal mesh width. In Table 4 we take $M=4 N$ to see the temporal errors. These numerical results show that the method (12) converges and the orders of convergence tend to 1 as $M$ and $N$ increase, which agrees with the error estimate of Theorem 1.

Table 3: Example 2: Maximum two-mesh nodal differences and orders of convergence

\begin{tabular}{|c||c|c|c|c|c|}
\hline & $\mathrm{M}=32$ & $\mathrm{M}=64$ & $\mathrm{M}=128$ & $\mathrm{M}=256$ & $\mathrm{M}=512$ \\
& $\mathrm{~N}=128$ & $\mathrm{~N}=256$ & $\mathrm{~N}=512$ & $\mathrm{~N}=1024$ & $\mathrm{~N}=2048$ \\
\hline$\alpha=1.2$ & $1.174 \mathrm{E}-01$ & $6.370 \mathrm{E}-02$ & $3.326 \mathrm{E}-02$ & $1.701 \mathrm{E}-02$ & $8.602 \mathrm{E}-03$ \\
& 0.882 & 0.937 & 0.968 & 0.983 & \\
\hline$\alpha=1.4$ & $4.913 \mathrm{E}-02$ & $2.627 \mathrm{E}-02$ & $1.365 \mathrm{E}-02$ & $6.977 \mathrm{E}-03$ & $3.534 \mathrm{E}-03$ \\
& 0.903 & 0.945 & 0.968 & 0.981 & \\
\hline$\alpha=1.6$ & $2.435 \mathrm{E}-02$ & $1.325 \mathrm{E}-02$ & $7.016 \mathrm{E}-03$ & $3.650 \mathrm{E}-03$ & $1.877 \mathrm{E}-03$ \\
& 0.877 & 0.918 & 0.943 & 0.959 & \\
\hline$\alpha=1.8$ & $1.043 \mathrm{E}-02$ & $5.905 \mathrm{E}-03$ & $3.247 \mathrm{E}-03$ & $1.749 \mathrm{E}-03$ & $9.287 \mathrm{E}-04$ \\
& 0.820 & 0.863 & 0.892 & 0.913 & \\
\hline \hline
\end{tabular}

The computed solution $\left\{u_{m}^{n}\right\}_{m=0, n=0}^{M, N}$ for $N=M=64$ and $\alpha=1.2,1.6$ is shown in Figure 1; it does not exhibit steep gradients, which agrees with our assumption (17) that $u_{t}$ and $u_{x}$ are bounded. In Figure 2 the discrete space derivative $D_{x}^{-} u_{m}^{n}$ for $N=M=64$ is shown and a singularity near $x=0$ is observed, which is consistent with our implicit assumption in (17) that $u_{x x}(x, t)$ blows up at $x=0$.

In the final example we illustrate Lemma 2 by showing convergence to a steady- 
Table 4: Example 2: Maximum two-mesh nodal differences and orders of convergence

\begin{tabular}{|c||c|c|c|c|c|}
\hline & $\mathrm{M}=64$ & $\mathrm{M}=128$ & $\mathrm{M}=256$ & $\mathrm{M}=512$ & $\mathrm{M}=1024$ \\
& $\mathrm{~N}=16$ & $\mathrm{~N}=32$ & $\mathrm{~N}=64$ & $\mathrm{~N}=128$ & $\mathrm{~N}=256$ \\
\hline$\alpha=1.2$ & $1.394 \mathrm{E}-01$ & $9.233 \mathrm{E}-02$ & $5.444 \mathrm{E}-02$ & $2.984 \mathrm{E}-02$ & $1.566 \mathrm{E}-02$ \\
& 0.594 & 0.762 & 0.867 & 0.930 & \\
\hline$\alpha=1.4$ & $7.552 \mathrm{E}-02$ & $4.846 \mathrm{E}-02$ & $2.790 \mathrm{E}-02$ & $1.511 \mathrm{E}-02$ & $7.893 \mathrm{E}-03$ \\
& 0.640 & 0.796 & 0.885 & 0.937 & \\
\hline$\alpha=1.6$ & $5.099 \mathrm{E}-02$ & $3.066 \mathrm{E}-02$ & $1.731 \mathrm{E}-02$ & $9.272 \mathrm{E}-03$ & $4.811 \mathrm{E}-03$ \\
& 0.734 & 0.824 & 0.901 & 0.947 & \\
\hline$\alpha=1.8$ & $3.737 \mathrm{E}-02$ & $2.237 \mathrm{E}-02$ & $1.241 \mathrm{E}-02$ & $6.568 \mathrm{E}-03$ & $3.389 \mathrm{E}-03$ \\
& 0.741 & 0.850 & 0.918 & 0.955 & \\
\hline \hline
\end{tabular}

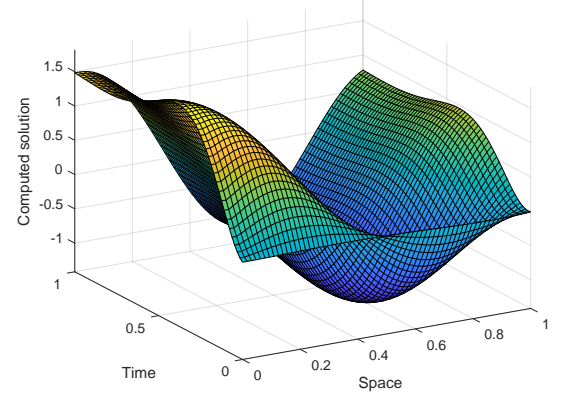

(a) $\alpha=1.2$

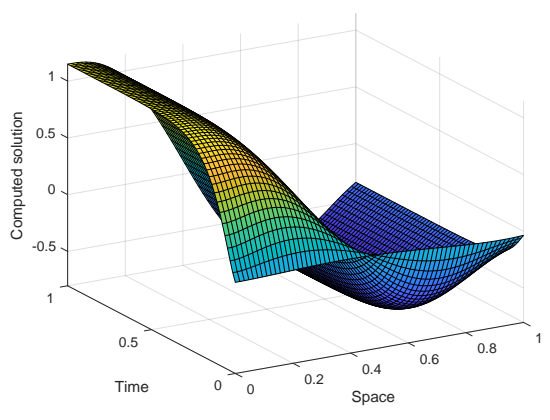

(b) $\alpha=1.6$

Figure 1: Example 2: Computed solutions with the method (12) for $N=M=64$ and $\alpha=1.2,1.6$.

state solution.

Example 3. Consider the problem

$$
\begin{aligned}
u_{t}-D_{R L C, x}^{\alpha} u & =0 \text { for }(x, t) \in(0,1) \times(0,1], \\
u(x, 0) & =50\left(x^{4}+r x^{3}+s x^{2}\right) \text { for } x \in(0,1), \\
D_{C, x}^{\alpha-1} u(0, t) & =D_{C, x}^{\alpha-1} u(1, t)=0 \text { for } t \in(0,1],
\end{aligned}
$$

where the constants $r$ and $s$ are chosen so that

$$
D_{C, x}^{\alpha-1} u(0,0)=D_{C, x}^{\alpha-1} u(1,0)=0 \text { and } \int_{x=0}^{1} u(x, 0)=0 .
$$




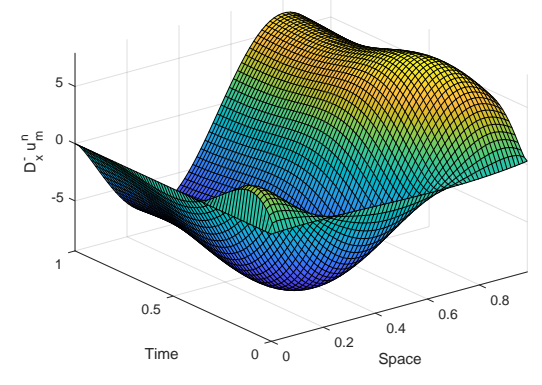

(a) Whole domain

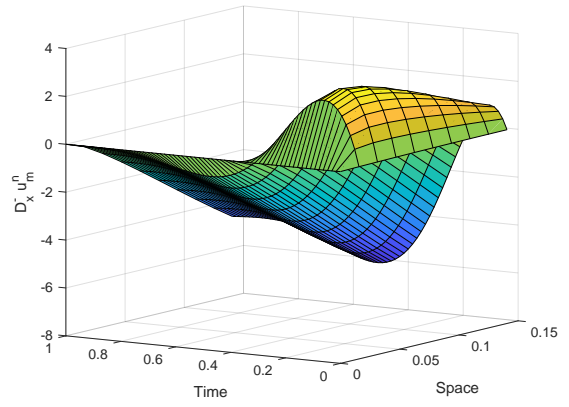

(b) Zoom near $x=0$

Figure 2: Example 2: Discrete spacial derivative for $N=M=64$ and $\alpha=1.2$ in the whole domain (left figure), with a zoom near $x=0$ (right figure).

That is,

$$
r=\frac{4(4-\alpha)}{\alpha}\left(\frac{1}{5}-\frac{4}{(5-\alpha)(4-\alpha)}\right) \text { and } s=-3\left(\frac{1}{5}+\frac{r}{4}\right) .
$$

In Table 5 we give the two-mesh differences $D_{M, N}$ and the orders of convergence $q_{M, N}$ for $\alpha=1.2,1.4,1.6$ and 1.8 with $N=M$. The computed orders of converge again agree with Theorem 1.

Table 5: Example 3: Maximum two-mesh nodal differences and orders of convergence

\begin{tabular}{|c||c|c|c|c|c|}
\hline & $\mathrm{M}=32$ & $\mathrm{M}=64$ & $\mathrm{M}=128$ & $\mathrm{M}=256$ & $\mathrm{M}=512$ \\
& $\mathrm{~N}=32$ & $\mathrm{~N}=64$ & $\mathrm{~N}=128$ & $\mathrm{~N}=256$ & $\mathrm{~N}=512$ \\
\hline$\alpha=1.2$ & $1.191 \mathrm{E}-01$ & $7.605 \mathrm{E}-02$ & $4.522 \mathrm{E}-02$ & $2.546 \mathrm{E}-02$ & $1.375 \mathrm{E}-02$ \\
& 0.647 & 0.750 & 0.829 & 0.888 & \\
\hline$\alpha=1.4$ & $8.820 \mathrm{E}-02$ & $5.416 \mathrm{E}-02$ & $3.107 \mathrm{E}-02$ & $1.694 \mathrm{E}-02$ & $8.920 \mathrm{E}-03$ \\
& 0.704 & 0.801 & 0.876 & 0.925 & \\
\hline$\alpha=1.6$ & $7.966 \mathrm{E}-02$ & $4.855 \mathrm{E}-02$ & $2.757 \mathrm{E}-02$ & $1.492 \mathrm{E}-02$ & $7.824 \mathrm{E}-03$ \\
& 0.714 & 0.816 & 0.886 & 0.931 & \\
\hline$\alpha=1.8$ & $8.438 \mathrm{E}-02$ & $5.113 \mathrm{E}-02$ & $2.880 \mathrm{E}-02$ & $1.552 \mathrm{E}-02$ & $8.112 \mathrm{E}-03$ \\
& 0.723 & 0.828 & 0.892 & 0.936 & \\
\hline \hline
\end{tabular}

In Figure 3 we display the computed solutions with the method (12) for $N=$ $M=64$ and $\alpha=1.2,1.4,1.6,1.8$. For each value of $\alpha$, it is clear that for $t$ sufficiently large, the steady-state solution $u(\cdot, t) \equiv 0$ predicted by Lemma 2 is approached. This steady-state solution conserves the mass of the initial condition. 


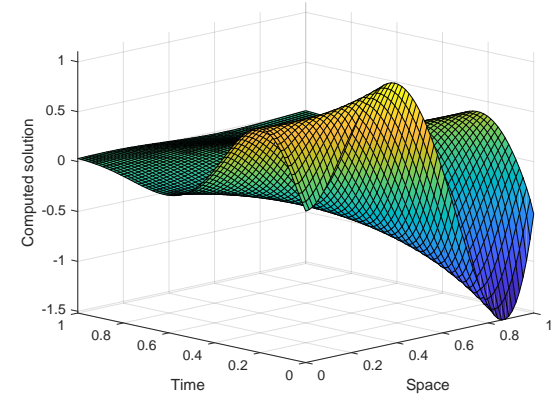

(a) $\alpha=1.2$

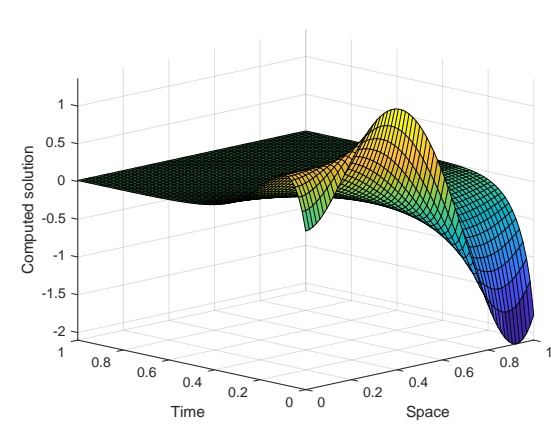

(c) $\alpha=1.6$

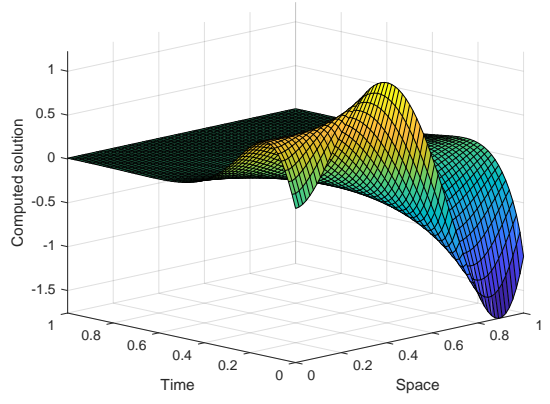

(b) $\alpha=1.4$

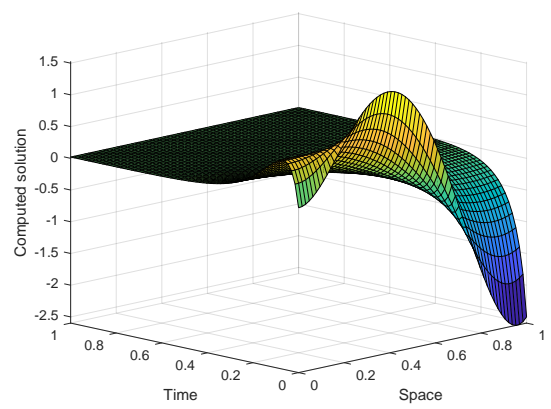

(d) $\alpha=1.8$

Figure 3: Example 3: Computed solutions with the method (12) for $N=M=64$ and $\alpha=$ 1.2, 1.4, 1.6, 1.8.

For each $n \in\{0,1, \ldots, N\}$, we approximate $\left\|u\left(\cdot, t_{n}\right)\right\|_{L^{2}(0,1)}$ by the composite trapezoidal rule on $\left\{x_{m}\right\}_{m=1}^{M}$, using the computed values $\left\{u_{m}^{n}\right\}_{m=1}^{M}$. These approximate values of $\left\|u\left(\cdot, t_{n}\right)\right\|_{L^{2}(0,1)}$ are given in Figure 4 and it is observed that the smaller $\alpha$ is, the later the steady state is reached; this agrees with our Remark 4.

\section{Acknowledgements}

The research of José Luis Gracia is partly supported by the Institute of Mathematics and Applications (IUMA), the project MTM2016-75139-R and the Diputación General de Aragón (E24-17R). The research of Martin Stynes is partly supported by the National Natural Science Foundation of China under grant NSAF-U1930402.

\section{Declarations of interest}

None. 


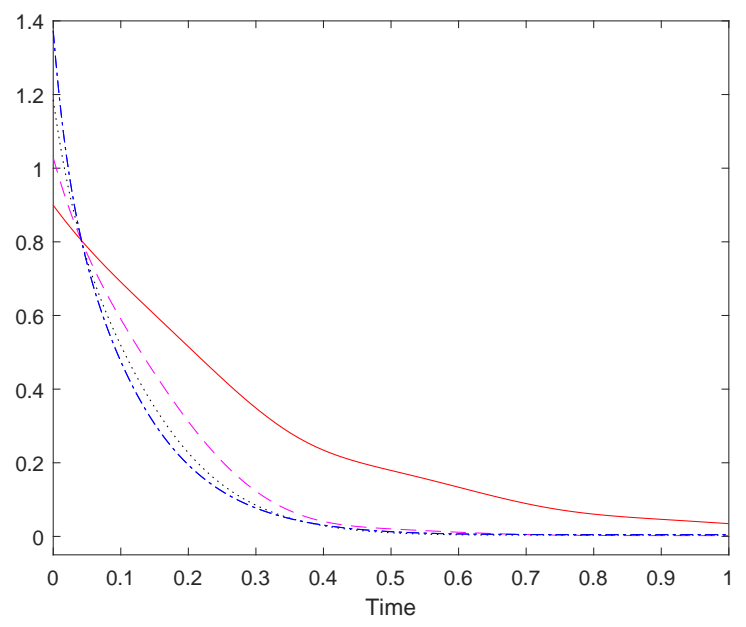

Figure 4: Example 3: Approximated values of $\left\|u\left(\cdot, t_{n}\right)\right\|_{L^{2}(0,1)}$ using (12) with $N=M=256$ and $\alpha=1.2$ (solid line), $\alpha=1.4$ (dashed line), $\alpha=1.6$ (dotted line) and $\alpha=1.8$ (dash-dotted line).

\section{References}

[1] Boris Baeumer, Mihály Kovács, Mark M. Meerschaert, and Harish Sankaranarayanan. Boundary conditions for fractional diffusion. J. Comput. Appl. Math., 336:408-424, 2018.

[2] Boris Baeumer, Mihály Kovács, and Harish Sankaranarayanan. Fractional partial differential equations with boundary conditions. J. Differential Equations, 264(2):1377-1410, 2018.

[3] Diego del Castillo-Negrete. Fractional diffusion models of nonlocal transport. Phys. Plasmas, 13(082308), 2006.

[4] Kai Diethelm. The analysis of fractional differential equations, volume 2004 of Lecture Notes in Mathematics. Springer-Verlag, Berlin, 2010. An application-oriented exposition using differential operators of Caputo type.

[5] V. J. Ervin, N. Heuer, and J. P. Roop. Regularity of the solution to 1-D fractional order diffusion equations. Math. Comp., 87(313):2273-2294, 2018.

[6] Vincent J. Ervin and John Paul Roop. Variational formulation for the stationary fractional advection dispersion equation. Numer. Methods Partial Differential Equations, 22(3):558-576, 2006. 
[7] Paul A. Farrell, Alan F. Hegarty, John J. H. Miller, Eugene O'Riordan, and Grigorii I. Shishkin. Robust computational techniques for boundary layers, volume 16 of Applied Mathematics (Boca Raton). Chapman \& Hall/CRC, Boca Raton, FL, 2000.

[8] José Luis Gracia, Eugene O'Riordan, and Martin Stynes. Convergence analysis of a finite difference scheme for a two-point boundary value problem with a Riemann-Liouville-Caputo fractional derivative. BIT. (To appear).

[9] K. Q. Lan and W. Lin. Positive solutions of systems of Caputo fractional differential equations. Commun. Appl. Anal., 17(1):61-85, 2013.

[10] Kassem Mustapha and Dominik Schötzau. Well-posedness of $h p$-version discontinuous Galerkin methods for fractional diffusion wave equations. IMA J. Numer. Anal., 34(4):1426-1446, 2014.

[11] Hans-Görg Roos, Martin Stynes, and Lutz Tobiska. Robust numerical methods for singularly perturbed differential equations, volume 24 of Springer Series in Computational Mathematics. Springer-Verlag, Berlin, second edition, 2008. Convection-diffusion-reaction and flow problems.

[12] Martin Stynes and José Luis Gracia. A finite difference method for a twopoint boundary value problem with a Caputo fractional derivative. IMA J. Numer. Anal., 35(2):698-721, 2015.

[13] Martin Stynes, Eugene O'Riordan, and José Luis Gracia. Error analysis of a finite difference method on graded meshes for a time-fractional diffusion equation. SIAM J. Numer. Anal., 55(2):1057-1079, 2017.

[14] Richard S. Varga. Matrix iterative analysis, volume 27 of Springer Series in Computational Mathematics. Springer-Verlag, Berlin, expanded edition, 2000 . 